\section{JTI}

JOURNAL OF

TRAUMA AND INJURY

\title{
Part 2. Clinical Practice Guideline for Trauma Team Composition and Trauma Cardiopulmonary Resuscitation from the Korean Society of Traumatology
}

Received: June 17, 2020

Accepted: June 23, 2020

\section{Correspondence to}

Gil Jae Lee, M.D., Ph.D.

Department of Traumatology, College of Medicine, Gachon University, Department of Trauma Surgery, Gachon University Gil Medical Center, 783 Namdong-daero, Namdong-gu, Incheon 21556, Korea

Tel: $+82-32-460-3010$

Fax: +82-32-460-2372

E-mail: nonajugi@gilhospital.com
Oh Hyun Kim, M.D., Ph.D. ${ }^{1}$, Seung Je Go, M.D. ${ }^{2}$, Oh Sang Kwon, M.D. ${ }^{3}$, Chan-Yong Park, M.D., Ph.D. ${ }^{4}$, Byungchul Yu, M.D. ${ }^{5}$, Sung Wook Chang, M.D. ${ }^{6}$, Pil Young Jung, M.D., Ph.D. ${ }^{7}$, Gil Jae Lee, M.D., Ph.D. ${ }^{5}$, KST (Korean Society of Traumatology) Clinical Research Group

${ }^{1}$ Department of Emergency Medicine, Wonju College of Medicine, Yonsei University, Wonju, Korea

${ }^{2}$ Department of Trauma Surgery, Chungbuk National University Hospital, Cheongju, Korea

${ }^{3}$ Department of Trauma Surgery, Cheju Halla General Hospital, Jeju, Korea

${ }^{4}$ Department of Trauma Surgery, Wonkwang University Hospital, Iksan, Korea

${ }^{5}$ Department of Traumatology, College of Medicine, Gachon University, Department of

Trauma Surgery, Gachon University Gil Medical Center, Incheon, Korea

${ }^{6}$ Department of Thoracic and Cardiovascular Surgery, Trauma Center, Dankook University Hospital, Cheonan, Korea

${ }^{7}$ Department of Surgery, Wonju Severance Christian Hospital, Yonsei University Wonju College of Medicine, Wonju, Korea

Based on the revised recommendations, the final recommendations were confirmed after collecting opinions from trauma experts, experts from the Korean Society of Traumatology, and research method experts using the Delphi technique (Table 1).

\section{SHOULD A TRAUMA UNIT BE ESTABLISHED IN ORDER TO TREAT TRAUMA PATIENTS?}

\section{Recommendation}

A. A trauma unit must be established in order to treat severe trauma patients (1C).

B. The trauma unit should be activated to respond within an appropriate time relative to the scale of the medical institution (1C).
This is an Open Access article distributed under the terms of the Creative Commons Attribution Non-Commercial License (http://creativecommons.org/licenses/by-nc/4.0/) which permits unrestricted noncommercial use, distribution, and reproduction in any medium, provided the original work is properly cited. 


\section{Evidence summary}

\section{Configuration of trauma units}

It is internationally understood and established that different medical specialists and personnel must work together to treat polytrauma patients. In many developed countries with trauma units, the completion of a professional trauma program such as Advanced Trauma Life Support, the Japan Advanced Trauma Evaluation and Care, or the European Trauma Course is required to join a trauma unit. Multiple studies have reported that the establishment of specialized trauma units has many advantages [1-4]. However, no study has evaluated the most appropriate configuration of the trauma unit. The membership of a trauma unit depends on the location and institution-specific circumstances [5-11]. Although some studies have reported that only two doctors are needed to effectively treat trauma patients $[12,13]$, other studies have reported the need to have more than two doctors in the trauma unit depending on the severity of the patient. The interpretation, experience, and education of each trauma unit differ according to local circumstances. It is stated in South Korea's administrative guideline for trauma centers that a trauma unit must be able to respond within 10 minutes and must consist of general surgeons, thoracic surgeons, neurosurgeons, and emergency medicine physicians. However, only 16 trauma centers meet these criteria, and most emergency departments in South Korea do not satisfy these criteria.

\section{The role and qualifications of the team leader}

The role of the team leader is to assess patients' data, con-

Table 1. Scores from the Delphi technique (Likert scale 1-9)

\begin{tabular}{|c|c|c|c|c|c|}
\hline \multicolumn{2}{|c|}{ Key question } & \multicolumn{2}{|r|}{ Recommendation } & \multirow{2}{*}{$\frac{\text { Mean }}{8.1}$} & \multirow{2}{*}{$\frac{\text { SD }}{1.5}$} \\
\hline 1 & Should a trauma unit be estab- & A & A trauma unit should be established in order to treat severe trauma patients (1C). & & \\
\hline & $\begin{array}{l}\text { lished in order to treat trauma } \\
\text { patients? }\end{array}$ & $B$ & $\begin{array}{l}\text { The trauma unit should be activated to respond within an appropriate time } \\
\text { relative to the scale of the medical institution (1C). }\end{array}$ & 8.3 & 1.6 \\
\hline 2 & $\begin{array}{l}\text { Should activation of the trauma } \\
\text { unit be stratified depending on } \\
\text { the severity of the trauma injury? }\end{array}$ & A & $\begin{array}{l}\text { A stratified response system maximizes medical resources and thus should be } \\
\text { considered ( } 2 \mathrm{~B}) \text {. }\end{array}$ & 7.5 & 1.2 \\
\hline 3 & $\begin{array}{l}\text { What are appropriate physiological } \\
\text { criteria for trauma unit activation? }\end{array}$ & $A$ & $\begin{array}{l}\text { The physiological criteria for trauma unit activation are the following (1B): } \\
\text { A. Respiratory rate }<10 \text { or }>30 \text { breaths per minute } \\
\text { B. Systolic blood pressure }<90 \mathrm{mmHg} \\
\text { C. Heart rate }>100 \text { bpm } \\
\text { D. GCS }<13\end{array}$ & 6.4 & 2.3 \\
\hline \multirow[t]{2}{*}{4} & \multirow{2}{*}{$\begin{array}{l}\text { What deceased should be made } \\
\text { when CPR is being performed in } \\
\text { trauma patients? }\end{array}$} & A & $\begin{array}{l}\text { Cardiac resuscitation must follow the standard cardiac resuscitation guideline } \\
(1 \mathrm{~A}) \text {. }\end{array}$ & 8.0 & 2.0 \\
\hline & & $B$ & $\begin{array}{l}\text { When CPR is being performed, the correction of reversible causes of cardiac } \\
\text { arrest should be performed regularly (1B). }\end{array}$ & 8.6 & 0.5 \\
\hline \multirow[t]{2}{*}{5} & \multirow[t]{2}{*}{$\begin{array}{l}\text { What indicators should be used } \\
\text { to monitor trauma patients with } \\
\text { cardiac arrest? }\end{array}$} & $A$ & $\begin{array}{l}\text { When a trauma patient is being treated with cardiac resuscitation, end-tidal } \mathrm{CO}_{2} \\
\text { is recommended to be used as an indicator to decide whether to terminate } \\
\text { resuscitation treatment and to predict the patient's death (1B). }\end{array}$ & 7.2 & 2.3 \\
\hline & & $B$ & $\begin{array}{l}\text { Although arterial cannulation can objectively measure the efficiency of the } \\
\text { CPR being performed and help diagnose cardiac arrest, cannulation must not } \\
\text { delay or stop the process of CPR ( } 2 \text { C). }\end{array}$ & 7.1 & 1.6 \\
\hline \multirow[t]{2}{*}{6} & \multirow{2}{*}{$\begin{array}{l}\text { What situations require CPR to } \\
\text { be initiated and terminated for } \\
\text { trauma patients? }\end{array}$} & A & $\begin{array}{l}\text { Termination of CPR must be considered when resuscitation is not successful } \\
\text { after the reversible cause of the cardiac arrest is corrected (1C). }\end{array}$ & 7.5 & 1.9 \\
\hline & & $B$ & $\begin{array}{l}\text { CPR termination must be considered if the patient is determined to have died } \\
\text { or exhibits signs and injuries that are unlikely to be survivable (1C). }\end{array}$ & 7.8 & 1.8 \\
\hline
\end{tabular}

SD: standard deviation, GCS: Glasgow coma scale, CPR: cardiopulmonary resuscitation, MTP: massive transfusion protocol. 
sider multiple treatment options, and swiftly decide on a treatment plan. Leadership requires the ability to facilitate communication among members and to work out an interdisciplinary agreement. Hoff et al. [14] reported that the implementation of a team leader led to improvements in the treatment process of a trauma patient. Furthermore, Alberts et al. [15] reported that after the implementation of a "trauma unit leader", the outcomes of trauma patients improved. The team leader must have superb knowledge regarding the treatment of polytrauma patients and experience in interdisciplinary treatment, which consists of being able to assess the patient depending on the situation, treat and monitor, consult with other medical specialties, adjust the medical team, and explain the decision process in the trauma unit to the patient's legal guardian. It is important that the interdisciplinary method of treatment does not cause a lag in the treatment process, which underscores the importance of establishing a treatment guideline $[14,16,17]$. According to a nationwide survey of trauma centers conducted by the American College of Surgeons Committee on Trauma (ACSCOT), onefourth of team leaders were trauma surgeons and one-half were general surgeons [17]. Although it was reported that the time to surgery was faster when the team leader was a trauma surgeon, there was no significant difference in survival outcomes [18]. The main role of the team leader is to manage the trauma unit. The management of a team involves recording the team members' competencies, and researching and making decisions at every step of diagnosis and treatment through discussion and agreement. In trauma, a team leader must possess the ability and qualifications to follow standard guidelines through an interdisciplinary approach to medicine [19].

\section{Response time}

The response time is defined as the time from the patient's arrival to the initiation of treatment by the trauma team doctor [20]. The response time allowed for level I and II trauma centers is 15 minutes, whereas for level III and IV trauma centers, the maximum allowed response time is 30 minutes. According to a study conducted by the Trauma Audit and Research Network, the inclusion of a trauma surgeon significantly reduced the mortality rate from $60 \%$ to $32 \%$ for trauma patients [21]. Luchette et al. [22] reported that when a trauma doctor resides in the hospital, the diagnosis was quicker and the operation was also faster. However, in cases of severe thoracic and head trauma, the time to intensive care treatment was not affected [1,2,10]. Wyatt et al. [23] reported that when an experienced doctor treated severe trauma patients $(1,427$ patients, injury severity score [ISS] $>15$ ), the survival rate was higher than when doctors without much experience treated these patients. It is also recommended that acute trauma patients be reevaluated within 24 hours of their initial treatment in order to prevent additional complications.

Although trauma centers in South Korea are not graded, the 16 trauma centers that exist function as level I centers. Therefore, it was determined that this clinical practical guideline was acceptable and applicable within South Korea.

\section{SHOULD ACTIVATION OF THE TRAUMA UNIT BE STRATIFIED DEPENDING ON THE SEVERITY OF THE TRAUMA INJURY?}

\section{Recommendation}

A stratified response system maximizes medical resources and thus should be considered (2B).

\section{Evidence summary}

\section{Activation of the trauma unit}

In an effective trauma system, the patient's status and severity must be determined and the patient must be transported prior to activating the trauma unit. Not only does the matrix triage system provide a more efficient way to treat severe trauma patients, but it also reduces transportation costs. The American Trauma Society states that $25 \%$ to $35 \%$ patients are overtriaged, whereas $5 \%$ are undertriaged [20]. However, when the sensitivity of the goal was set to $80 \%$, a study was not able to reduce the ratio of underestimation to lower than 70\% [24]. Accordingly, the ACSCOT responded by publishing a standard matrix triage system, which has proven to be more efficient. A study utilizing the ACSCOT matrix triage system conducted at a level I trauma center with 7,031 trauma 
patients reported that matrix triage system yielded a $45 \%$ rate of overestimation and a $24 \%$ rate of underestimation [25]. Since a stratified medical system maximizes medical resources, the application of such implementation of such systems may be considered for South Korea.

\section{WHAT ARE APPROPRIATE PHYSIOLOGI- CAL CRITERIA FOR TRAUMA UNIT ACTI- VATION?}

\section{Recommendation}

The physiological criteria for trauma unit activation are the following (1B):
A. Respiratory rate $<10$ or $>30$ breaths per minute
B. Systolic blood pressure $<90 \mathrm{mmHg}$
C. Heart rate $>100 \mathrm{bpm}$
D. Glasgow coma scale $(\mathrm{GCS})<13$

The criteria for trauma unit activation according to the 2016 operating instructions for trauma centers in South Korea are as follows:

1. Physiological indications

A. Airway obstruction/Decreased respiratory rate

B. Intubation prior to arrival

C. Adults: Respiratory rate $<10$ or $>30$ breaths per minute

D. Adults: Systolic blood pressure $<90 \mathrm{mmHg}$

E. Adults: heart rate $>100 \mathrm{bpm}$

F. GCS $<13$

G. A transferred patient undergoing blood transfusion to maintain vital signs

$\mathrm{H}$. Worsening status of a stabilized patient

2. Anatomical criteria

A. Penetration injury

i. Penetrating injury to the craniocervical, thoracic, or abdominal region

ii. Limbs: Penetrating injury proximal to the elbow or knee

B. Thorax

i. Chest wall instability or deformity (e.g. flail chest)
C. Nervous system

i. Open or depressed skull fracture

ii. Quadriplegia or suspicion of spine injury

D. Orthopedic

i. Pelvic fracture

ii. Two or more proximal long-bone fractures

iii. Crushed, degloved, mangled, or pulseless extremity

iv. Amputation proximal to wrist or ankle

\section{Mechanism of injury}

A. Death in the same passenger compartment

B. Ejection (partial or complete) from an automobile

C. Traffic collision at over $60 \mathrm{~km} / \mathrm{h}$

D. Automobile versus pedestrian/bicyclist: thrown, run over, or with significant (over $30 \mathrm{~km} / \mathrm{h}$ ) impact

E. Rescue time exceeding 20 minutes

F. Motorcycle, bicycle, etc.: collision over $30 \mathrm{~km} / \mathrm{h}$ or roll-over accident

G. Fall greater than or equal to: $6 \mathrm{~m}$ for adults, $3 \mathrm{~m}$ for children

H. Injury from an explosion

※ When deemed necessary by a clinician in the resuscitation area.

\section{Evidence summary}

\section{Blood pressure and respiratory rate}

Low blood pressure in trauma patients is correlated with a 1.6-fold increase in emergency operations, a 24 -fold increase in the mortality rate, and a 7-fold increase in the need for intensive care unit (ICU) treatment [26]. The ACSCOT implements low blood pressure as a criteiron for transporting the patient to the trauma center, whereas hospitals in the state of South Wales in Australia use blood pressure as an indicator for activating the trauma unit $[20,27]$. Furthermore, in a study conducted by New York's trauma registry, low blood pressure and a respiratory rate of $<10 / \mathrm{min}$ or $>29 /$ min were correlated with mortality rates of $32.9 \%$ and $28.8 \%$, respectively [28].

\section{GCS}

According to Kohn et al. [29], 44.2\% patients with a GCS score of less than 10 were confirmed to have severe brain 
injury. Although a GCS score of less than 14 was shown to be correlated with the presence of a pathological brain injury [30,31], the need to activate the trauma unit was not implicit (GCS $\leq 14$ and $\geq 11$ ). In another study, a GCS score less than 10 was correlated with a 3.5-fold increase in mortality during surgery [32]. In the ACSCOT guideline, the indications for trauma activation include a car accident that caused the car to be dented by more than 20-30 inches, a pedestrian hit at the speed of 20-30 $\mathrm{mph}$, or a passenger dying from the traffic accident [20]. It is also stated that a rollover car crash increases the risk of severe trauma [33].

\section{Mechanism of injury}

When the trauma unit was activated based on the mechanism of injury alone, $92 \%$ of cases were overestimated, and the sensitivity and PPV were reported to be $50-70 \%$ and $16.1 \%$, respectively [34-36]. However, when the mechanism of injury was included in the criteria, the sensitivity and specificity increased to $80 \%$ and $90 \%$, respectively [35]. Kohn et al. [29] reported in a prospective study that $9.4 \%$ of patients who fell from a height over 6 $\mathrm{m}$ needed ICU care or immediate surgery. Furthermore, Yagmur et al. [37] reported that patients who died from falling injuries fell from heights over $9 \mathrm{~m}$.

\section{Age}

When Kohn et al. [29] analyzed various trauma activation protocols, such as that of the ACSCOT, it was determined that an age cut-off of over 65 years was most appropriate for activating the trauma unit [29]. Demetriades et al. [38] reported that patients over 70 years of age had a $16 \%$ higher mortality rate and a 19\% higher likelihood of needing surgical and ICU treatment than younger patients. Mackenzie demonstrated that after the age of 55 years, the risk of fatal trauma injuries significantly increased [39]. Lastly, Grossman et al. [40] reported that patients over the age of 65 had a $6.8 \%$ higher mortality rate than their younger counterparts. Since, South Korea uses most of the Centers for Disease Control and Prevention field triage guidelines, it was determined that this clinical practical guideline was applicable within South Korea.

\section{WHAT FACTORS SHOULD BE CONSID- ERED WHEN CARDIOPULMONARY RESUSCITATION (CPR) IS BEING PER- FORMED IN TRAUMA PATIENTS?}

\section{Recommendation}

A. Cardiac resuscitation must follow the standard cardiac resuscitation guideline (1A).

$\mathrm{B}$. When CPR is being performed, the correction of reversible causes of cardiac arrest should be performed regularly (1B).

\section{Evidence summary}

According to the International Liaison Committee on Resuscitation (ILCOR) and the European Resuscitation Council (ERC) guideline, trauma patients receiving cardiac resuscitation should be treated according to standard cardiac resuscitation guidelines [41-44]. The 2015 American and European cardiac resuscitation guidelines recommend that the $5 \mathrm{H}$ 's and 5 T's (hypovolemia, hypoxia, hydrogen ion [acidosis], hypo/hyperkalemia, hypothermia, tension pneumothorax, cardiac tamponade, coronary thrombosis, pulmonary thrombosis, toxins) or the $4 \mathrm{H}$ 's and 4 T's must be treated actively during cardiac resuscitation $[43,44]$.

Complications from hemorrhage such as severe brain injury and hypovolemia are the most common cause of cardiac arrest from trauma. Other common causes of traumatic cardiac arrest include other reversible causes such as hypoxia, tension pneumothorax, and tamponade, which must be corrected immediately [43]. Not only does the above guideline recommend assessing, diagnosing, and treating the causes of cardiac arrest of trauma patients as soon as possible, but it states that treating the reversible causes should be prioritized before performing cardiac compressions [43]. In trauma patients, the primary cause of cardiac arrest must be treated by rapidly administering crystalloids and utilizing surgical endoscopic and endovascular techniques to control hemorrhage [44].

Furthermore during the hands-off time of cardiac resuscitation, it is recommended that a trained sonographer perform heart sonography in order to diagnose and treat the cause of hypovolemic cardiac arrest [44]. Some retrospective studies have reported that inserting a chest tube 
for preventing or treating tension pneumothorax can increase the survival rate for trauma patients with cardiac arrest [45-48].

In the S3 guideline published by the German Trauma Society (DGU), 10,359 patients in the trauma registry were analyzed. In this study the survival rate of trauma-induced cardiac arrest patients was $7.8 \%$ when the patient was treated with thoracotomy at the emergency department. However, the type of trauma was associated with a difference in the survival rate: that of cardiac arrest caused by blunt trauma was $1.6 \%$, whereas that of cardiac arrest caused by penetrating trauma was $11.2 \%$ [49-51]. Lastly it is recommended by the EAST guideline that cardiac arrests caused by penetrating trauma injuries should be treated with emergency thoracotomy [52].

The DGU S3 guideline implemented much of the 2005 ERC guideline, and it was determined that a more updated guideline would be needed. In the ERC and AHA 2015 guideline, policies were implemented from the 2010 recommendations without further review. Since the ERC guideline presents a thorough algorithm for performing $\mathrm{CPR}$ in cases of trauma, it was determined that the cardiac arrest algorithm from the ERC guideline would be implemented (Fig. 1).

\section{WHAT INDICATORS SHOULD BE USED TO MONITOR TRAUMA PATIENTS WITH CARDIAC ARREST?}

\section{Recommendation}

A. When a trauma patient is being treated with cardiac resuscitation, end-tidal $\mathrm{CO}_{2}$ is recommended to be used as an indicator to decide whether to terminate resuscitation treatment and to predict the patient's death (1B).

B. Although arterial cannulation can objectively measure the efficiency of CPR and help diagnose cardiac arrest, cannulation must not delay or stop the process of CPR (2C).

\section{Evidence summary}

Physiological indicators can be helpful alongside clinical signs and electrocardiograms when performing CPR.
However, no recommendations exist regarding which specific physiological indicator(s) should be used to determine the need for CPR. This fact stems from the concern that the evidence used to determine the need for CPR could actually do more harm than good. End-tidal $\mathrm{CO}_{2}$ $\left(\mathrm{ETCO}_{2}\right)$ should not be used alone to predict death and the termination of CPR [46], but $\mathrm{ETCO}_{2}$ can be recommended for use as a threshold value [53].

The initiation time of CPR should be determined by the readings of an electrocardiogram and signs of cardiac arrest [54]. Pulse oximetry and capnography readings are essential for polytrauma patients and can be used during cardiac arrest. However, the limitations of such indicators for patients in shock or hypothermia should be noted [44]. To assess the quality of CPR, the intubation tube must be placed in the correct location, and waveform capnography can be used to monitor the return of spontaneous circulation [54].

According to the S3 guideline, early catheterization of the femoral artery can be used to continuously measure blood pressure, help diagnose cardiac arrest in the emergency room, and objectively measure the effectiveness of CPR. However, it was recommended that catheterization must not delay or stop the process of CPR [44].

In the ERC and AHA 2015 guidelines, policies were implemented from the 2010 recommendations without further review. In the DGU S3 guideline on cardiac resuscitation, much of the information was based on the ERC 2005 guideline. Therefore, these guidelines should be updated.

\section{WHAT SITUATIONS REQUIRE CPR TO BE INITIATED AND TERMINATED FOR TRAU- MA PATIENTS?}

\section{Recommendation}

A. Termination of CPR must be considered when resuscitation is not successful after the reversible cause of cardiac arrest is corrected (1C).

B. CPR termination must be considered if the patient is determined to have deceased or exhibits signs and injuries that are unlikely to be survivable (1C). 

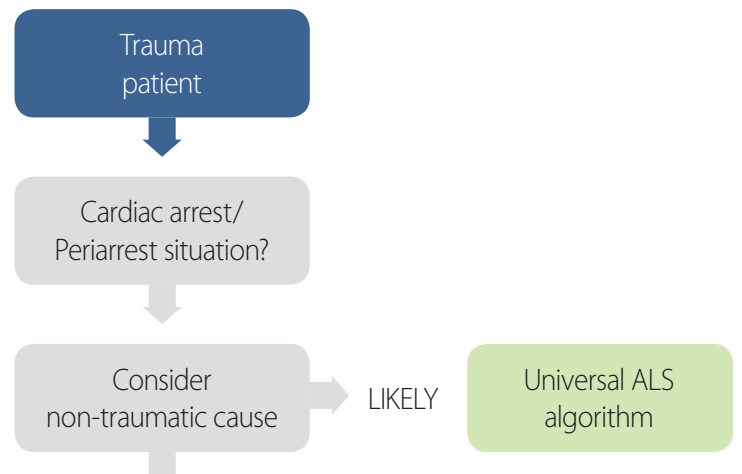

Hypoxia

Tension pneumothorax

Tamponade

Hypovolaemia
Consider termination of CPR

UNLIKELY

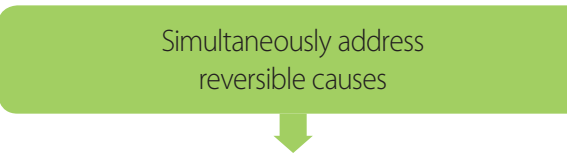

1. Control external catastrophic haemorrhage

2. Control airway and maximise oxygenation

3. Bilateral chest decompression

4. Relieve cardiac tamponade

5. Surgery for haemorrhage control

or proximal aortic compression

6. Massive transfusion protocol and fluids

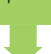

$\mathrm{NO}$

Return of spontaneous circulation
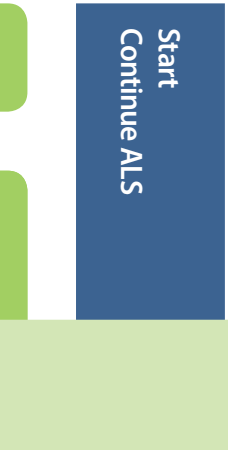

\section{YES}

Pre-hospital:

- Perform only life-saving interventions

- Immediate transport to appropriate hospital

In-hospital:

- Damage control resuscitation

- Definitive haemorrhage control

Fig. 1. Guideline for cardiac resuscitation in traumatic patients. Reprinted from Truhlár et al. ${ }^{45}$

\section{Evidence summary}

In a previous study conducted throughout the 1980s to the 1990s, the ERC reported that cardiac arrest trauma patients had a survival rate below $2 \%$. However, according to a German and American study conducted using cardiac arrest trauma patients from the DGU registry, the survival rate of such patients was $17.2 \%$ and $13 \%$ respec- tively, a higher rate than was previously thought $[55,56]$. It must be noted, though, that the American study only included cardiac arrest patients who arrived within the ICU bay within 1 hour.

If the health care provider is unable to find a pulse, or other clinical signs suggesting cardiac arrest, the health care provider must without hesitation start CPR. The clin- 
ical guideline for detecting cardiac arrest is no different in trauma than in other situations. The need for CPR should be determined according to the AHA or ERC guideline [57].

Determining the need for CPR by pulse must take no longer than 10 seconds. If this time is exceeded, CPR is performed whether a pulse was found or not. Simultaneously checking the absence of breathing and gasping, which occurs within 5 minutes in $40 \%$ of cardiac arrest patients, and the pulse of the patient is ideal so that the time to perform CPR is not postponed $[58,59]$. Furthermore, seizures must be recognized as a symptom of cardiac arrest [59].

The cessation of CPR is determined by the possibility of successful resuscitation or an underlying clinical judgment of a failed attempt at resuscitation. Furthermore, the cessation of CPR does not need the approval of the patient or caretaker; thus, if the caretaker demands CPR for a patient in whom it would be meaningless, the health care providers must explain that continuing CPR would be meaningless and cease performing CPR. The continuation of meaningless CPR can worsen the ability to make logical judgments and provide false hope for the patient and his or her caretaker. The decision to cease CPR should be explained as a means to stop causing further damage and to maximize the comfort and quality of life for the patient, instead of as a decision to give up [60].

Most initial deaths of polytrauma patients are caused by severe traumatic brain injury and massive hemorrhage. Furthermore, injuries without a possible survival outcome may occur (e.g., aortic injuries). The success of CPR depends on two factors: first, the time of performing CPR after cessation and second, the removal of a reversible cause of cessation of the heart. The typical reversible causes of traumatic cardiac arrest are shock caused by hemorrhage, tension pneumothorax, and cardiac tamponade. Some other causes such as severe brain damage and aortic injury are near-impossible to treat in the acute phase. Even if the cause of cardiac arrest is removed, CPR may fail, and CPR must be ceased if cardiac arrest continues and the cause of cardiac arrest cannot be found. The clinical sign of death must reflect irreversible death of essential organs at the cellular level and can be used as a marker for predicting the failure of CPR. If the patient dies, there is no possibility of survival, and there are clinical signs of cellular injury, CPR must be ceased. The decision to continue or cease CPR must be decided through the discussion of the medical staff involved in the process of CPR. There is no time indication for the failure of CPR. If the patient is eligible for organ donorship, once CPR is ceased, the patient can be considered for transportation to a medical facility for organ donation [60].

Although the American College of Surgeons and the National Association of EMS recommends the cessation of CPR for cardiac arrest trauma patients with no pulse, respiration, or detectable electrical pulses on an electrocardiogram [61], there have been cases where such patients have made full neurological recoveries. Thus, following these guidelines is recommended [62]:

In the following situations, CPR cessation for traumatic arrest should be considered:

- If after 15 minutes, no signs of life are detected

- For severe traumatic injuries (e.g., severe injuries such decapitation, penetrating cardiac injuries, and the outflow of brain tissue), where the likelihood of survival is low

- After a reversible cause of cardiac arrest is confirmed and corrected, but spontaneous circulation is not established.

- When cardiac activity cannot be seen on cardiac ultrasound.

It was determined that this clinical practical guideline is applicable within South Korea. Since the initiation and cessation of CPR faces many legal problems, making a decision at the prehospital phase is a hard task. However, this guideline considers traumatic cardiac arrest patients for in-hospital situations and was therefore determined to be applicable within South Korea.

\section{REFERENCES}

1. Dodek P, Herrick R, Phang PT. Initial management of trauma by a trauma team: effect on timeliness of care in a teaching hospital. Am J Med Qual 2000;15:3-8.

2. Vernon DD, Furnival RA, Hansen KW, Diller EM, Bolte RG, Johnson DG, et al. Effect of a pediatric trauma response team on emergency department treatment time and mortality of pe- 
diatric trauma victims. Pediatrics 1999;103:20-4.

3. Palmer SH, Maheson M. A radiological review of cervical spine injuries from an accident and emergency department: has the ATLS made a difference? J Accid Emerg Med 1995;12:189-90.

4. Lomas GA, Goodall O. Trauma teams vs non-trauma teams. Accid Emerg Nurs 1994;2:205-10.

5. Cornwell EE 3rd, Chang DC, Phillips J, Campbell KA. Enhanced trauma program commitment at a level I trauma center: effect on the process and outcome of care. Arch Surg 2003;138:838-43.

6. Eastes LS, Norton R, Brand D, Pearson S, Mullins RJ. Outcomes of patients using a tiered trauma response protocol. J Trauma 2001;50:908-13.

7. Demarest GB, Scannell G, Sanchez K, Dziwulski A, Qualls C, Schermer CR, et al. In-house versus on-call attending trauma surgeons at comparable level I trauma centers: a prospective study. J Trauma 1999;46:535-40; discussion 540-2.

8. Kaplan LJ, Santora TA, Blank-Reid CA, Trooskin SZ. Improved emergency department efficiency with a three-tier trauma triage system. Injury 1997;28:449-53.

9. Deo SD, Knottenbelt JD, Peden MM. Evaluation of a small trauma team for major resuscitation. Injury 1997;28:633-7.

10. Highley DA. Review of the composition and use of trauma teams within the Trent Region. J Accid Emerg Med 1994;11:1835.

11. Brennan R, Cohen SS, Chambers JA, Andrews C. The OR suite as a unique trauma resuscitation bay. AORN J 1994;60:576-7, $580-4$.

12. Carmody IC, Romero J, Velmahos GC. Day for night: should we staff a trauma center like a nightclub? Am Surg 2002;68:104851.

13. Allen DM, Hicks G, Bota GW. Outcomes after severe trauma at a northern Canadian regional trauma centre. Can J Surg 1998;41:53-8.

14. Hoff WS, Reilly PM, Rotondo MF, DiGiacomo JC, Schwab CW. The importance of the command-physician in trauma resuscitation. J Trauma 1997;43:772-7.

15. Alberts KA, Bellander BM, Modin G. Improved trauma care after reorganisation: a retrospective analysis. Eur J Surg 1999;165:426-30.

16. Hartmann J, Gabram S, Jacobs L, Libby M. A model for an integrated emergency medicine/trauma service. Acad Emerg Med 1996;3:1136-9.

17. Sugrue M, Seger M, Kerridge R, Sloane D, Deane S. A prospective study of the performance of the trauma team leader. J Trau- ma 1995;38:79-82.

18. Khetarpal S, Steinbrunn BS, McGonigal MD, Stafford R, Ney AL, Kalb DC, et al. Trauma faculty and trauma team activation: impact on trauma system function and patient outcome. J Trauma 1999;47:576-81.

19. Wurmb T, Balling H, Frühwald P, Keil T, Kredel M, Meffert R, et al. Polytrauma management in a period of change: time analysis of new strategies for emergency room treatment. Unfallchirurg 2009;112:390-9.

20. Rotondo M, Cribari C, Smith RS. Resources for the optimal care of the injured patient. Chicago:American College of Surgeons; 2014.

21. Lecky F, Woodford M, Yates DW. Trends in trauma care in England and Wales 1989-97. UK Trauma Audit and Research Network. Lancet 2000;355:1771-5.

22. Luchette F, Kelly B, Davis K, Johanningman J, Heink N, James L, et al. Impact of the in-house trauma surgeon on initial patient care, outcome, and cost. J Trauma 1997;42:490-5; discussion 495-7.

23. Wyatt JP, Henry J, Beard D. The association between seniority of Accident and Emergency doctor and outcome following trauma. Injury 1999;30:165-8.

24. Kane G, Wheeler NC, Cook S, Englehardt R, Pavey B, Green K, et al. Impact of the Los Angeles County Trauma System on the survival of seriously injured patients. J Trauma 1992;32:576-83.

25. Davis JW, Dirks RC, Sue LP, Kaups KL. Attempting to validate the overtriage/undertriage matrix at a level I trauma center. J Trauma Acute Care Surg 2017;83:1173-8.

26. Tinkoff GH, O'Connor RE. Validation of new trauma triage rules for trauma attending response to the emergency department. J Trauma 2002;52:1153-8; discussion 1158-9.

27. Smith J, Caldwell E, Sugrue M. Difference in trauma team activation criteria between hospitals within the same region. Emerg Med Australas 2005;17:480-7.

28. Henry MC. Trauma triage: New York experience. Prehosp Emerg Care 2006;10:295-302.

29. Kohn MA, Hammel JM, Bretz SW, Stangby A. Trauma team activation criteria as predictors of patient disposition from the emergency department. Acad Emerg Med 2004;11:1-9.

30. Kuhne CA, Ruchholtz S, Kaiser GM, Nast-Kolb D; Working Group on Multiple Trauma of the German Society of Trauma. Mortality in severely injured elderly trauma patients--when does age become a risk factor? World J Surg 2005;29:1476-82.

31. Norwood SH, McAuley CE, Berne JD, Vallina VL, Creath RG, 
McLarty J. A prehospital Glasgow Coma Scale score $<$ or $=14$ accurately predicts the need for full trauma team activation and patient hospitalization after motor vehicle collisions. J Trauma 2002;53:503-7.

32. Engum SA, Mitchell MK, Scherer LR, Gomez G, Jacobson L, Solotkin $\mathrm{K}$, et al. Prehospital triage in the injured pediatric patient. J Pediatr Surg 2000;35:82-7.

33. Champion HR, Lombardo LV, Shair EK. The importance of vehicle rollover as a field triage criterion. J Trauma 2009;67:350-7.

34. Norcross ED, Ford DW, Cooper ME, Zone-Smith L, Byrne TK, Yarbrough DR 3rd. Application of American College of Surgeons' field triage guidelines by pre-hospital personnel. J Am Coll Surg 1995;181:539-44.

35. Bond RJ, Kortbeek JB, Preshaw RM. Field trauma triage: combining mechanism of injury with the prehospital index for an improved trauma triage tool. J Trauma 1997;43:283-7.

36. Santaniello JM, Esposito TJ, Luchette FA, Atkian DK, Davis KA, Gamelli RL. Mechanism of injury does not predict acuity or level of service need: field triage criteria revisited. Surgery 2003;134:698-703; discussion 703-4.

37. Yagmur Y, Gülo lu C, Aldemir M, Orak M. Falls from flatroofed houses: a surgical experience of 1643 patients. Injury 2004;35:425-8.

38. Demetriades D, Sava J, Alo K, Newton E, Velmahos GC, Murray JA, et al. Old age as a criterion for trauma team activation. J Trauma 2001;51:754-6; discussion 756-7.

39. MacKenzie EJ, Rivara FP, Jurkovich GJ, Nathens AB, Frey KP, Egleston BL, et al. A national evaluation of the effect of trauma-center care on mortality. N Engl J Med 2006;354:366-78.

40. Grossman MD, Miller D, Scaff DW, Arcona S. When is an elder old? Effect of preexisting conditions on mortality in geriatric trauma. J Trauma 2002;52:242-6.

41. Nolan JP, Deakin CD, Soar J, Böttiger BW, Smith G. European Resuscitation Council guidelines for resuscitation 2005. Resuscitation 2005;67:S39-86.

42. Biarent D; International Liaison Committee on Resuscitation. 2005 international consensus on cardiopulmonary resuscitation and emergency cardiovascular care science with treatment recommendations. Circulation 2005;112:1-136.

43. Truhlá A, Deakin CD, Soar J, Khalifa GE, Alfonzo A, Bierens JJ, et al. European Resuscitation Council guidelines for resuscitation 2015: section 4. Cardiac arrest in special circumstances. Resuscitation 2015;95:148-201.

44. Neumar RW, Shuster M, Callaway CW, Gent LM, Atkins DL,
Bhanji F, et al. Part 1: executive summary: 2015 American Heart Association guidelines update for cardiopulmonary resuscitation and emergency cardiovascular care. Circulation 2015;132(18 suppl 2):S315-67.

45. Huber-Wagner S, Lefering R, Qvick M, Kay MV, Paffrath T, Mutschler W, et al. Outcome in 757 severely injured patients with traumatic cardiorespiratory arrest. Resuscitation 2007;75:276-85.

46. Mistry N, Bleetman A, Roberts KJ. Chest decompression during the resuscitation of patients in prehospital traumatic cardiac arrest. Emerg Med J 2009;26:738-40.

47. Bushby N, Fitzgerald M, Cameron P, Marasco S, Bystrzycki A, Rosenfeld JV, et al. Prehospital intubation and chest decompression is associated with unexpected survival in major thoracic blunt trauma. Emerg Med Australas 2005;17:443-9.

48. Irfan FB, Consunji R, El-Menyar A, George P, Peralta R, AlThani H, et al. Cardiopulmonary resuscitation of out-of-hospital traumatic cardiac arrest in Qatar: a nationwide population-based study. Int J Cardiol 2017;240:438-43.

49. Working Group, Ad Hoc Subcommittee on Outcomes, American College of Surgeons. Committee on Trauma. Practice management guidelines for emergency department thoracotomy. Working Group, Ad Hoc Subcommittee on Outcomes, American College of Surgeons-Committee on Trauma. J Am Coll Surg 2001;193:303-9.

50. Karmy-Jones R, Nathens A, Jurkovich GJ, Shatz DV, Brundage $S$, Wall MJ Jr, et al. Urgent and emergent thoracotomy for penetrating chest trauma. J Trauma 2004;56:664-8; discussion 668-9.

51. Powell DW, Moore EE, Cothren CC, Ciesla DJ, Burch JM, Moore $\mathrm{JB}$, et al. Is emergency department resuscitative thoracotomy futile care for the critically injured patient requiring prehospital cardiopulmonary resuscitation? J Am Coll Surg 2004;199:211-5.

52. Seamon MJ, Haut ER, Van Arendonk K, Barbosa RR, Chiu WC, Dente CJ, et al. An evidence-based approach to patient selection for emergency department thoracotomy: a practice management guideline from the Eastern Association for the Surgery of Trauma. J Trauma Acute Care Surg 2015;79:159-73.

53. Nolan JP, Hazinski MF, Aickin R, Bhanji F, Billi JE, Callaway CW, et al. Part 1: executive summary: 2015 international consensus on cardiopulmonary resuscitation and emergency cardiovascular care science with treatment recommendations. Resuscitation 2015;95:e1-31.

54. Williams ST, Sykes MC, Boon Lim P, Salciccioli JD. The 2015 advanced life support guidelines: a summary and evidence for 
the updates. Emerg Med J 2016;33:357-60.

55. Zwingmann J, Lefering R, Feucht M, Südkamp NP, Strohm PC, Hammer T. Outcome and predictors for successful resuscitation in the emergency room of adult patients in traumatic cardiorespiratory arrest. Critical Care 2016;20:282.

56. Ahmed N, Greenberg P, Johnson VM, Davis JM. Risk stratification of survival in injured patients with cardiopulmonary resuscitation within the first hour of arrival to trauma centre: retrospective analysis from the national trauma data bank. Emerg Med J 2017;34:282-8.

57. Soar J, Nolan JP, Böttiger BW, Perkins GD, Lott C, Carli P, et al. European Resuscitation Council guidelines for resuscitation 2015: section 3. Adult advanced life support. Resuscitation 2015;95:100-47.

58. Kleinman ME, Brennan EE, Goldberger ZD, Swor RA, Terry M, Bobrow BJ, et al. Part 5: adult basic life support and cardiopulmonary resuscitation quality: 2015 American Heart Association guidelines update for cardiopulmonary resuscitation and emergency cardiovascular care. Circulation 2015;132(18 suppl
2):S414-35.

59. Monsieurs KG, Nolan JP, Bossaert LL, Greif R, Maconochie IK, Nikolaou NI, et al. European Resuscitation Council guidelines for resuscitation 2015: section 1. Executive Summary. Resuscitation 2015;95:1-80.

60. Bossaert LL, Perkins GD, Askitopoulou H, Raffay VI, Greif R, Haywood KL, et al. European Resuscitation Council guidelines for resuscitation 2015: section 11. The ethics of resuscitation and end-of-life decisions. Resuscitation 2015;95:302-11.

61. Millin MG, Galvagno SM, Khandker SR, Malki A, Bulger EM, Standards and Clinical Practice Committee of the National Association of EMS Physicians (NAEMSP), et al. Withholding and termination of resuscitation of adult cardiopulmonary arrest secondary to trauma: resource document to the joint NAEMSP-ACSCOT position statements. J Trauma Acute Care Surg 2013;75:459-67.

62. Leis CC, Hernández CC, Blanco MJ, Paterna PC, Hernández Rde E, Torres EC. Traumatic cardiac arrest: should advanced life support be initiated? J Trauma Acute Care Surg 2013;74:634-8. 